high-TB prevalence areas. To eliminate TB in the future, the importance of anti-smoking campaigns should not be overlooked.

0 @ERSpublications

Current and ex-smoking is associated with increased risk of LTBI in high-risk individuals, especially in aged populations http://ow.ly/q5FTK

Jia-Yih Feng ${ }^{1,2}$, Shiang-Fen Huang ${ }^{1,3}$, Wen-Ying Ting ${ }^{1}$, Ming-Che Lee ${ }^{1}$, Yu-Chun Chen ${ }^{3,4}$, Yung-Yang Lin ${ }^{2,5,6}$, $\mathrm{Yu}$-Chin Lee ${ }^{1,3}$ and Wei-Juin $\mathrm{Su}^{1,3}$

${ }^{1}$ Dept of Chest Medicine, Taipei Veterans General Hospital, Taipei, ${ }^{2}$ Institute of Clinical Medicine, School of Medicine, National Yang-Ming University, Taipei, ${ }^{3}$ School of Medicine, National Yang-Ming University, Taipei, ${ }^{4}$ Dept of Medical Research and Education, National Yang-Ming University Hospital, Taipei, ${ }^{5}$ Institute of Brain Science, National YangMing University, Taipei, and ${ }^{6}$ Laboratory of Neurophysiology and Dept of Neurology, Taipei Veterans General Hospital, Taipei, Taiwan, R.O.C.

Correspondence: W-J. Su, Dept of Chest Medicine, Taipei Veterans General Hospital, \#201, Section 2, Shih-Pai Road, Taipei 112, Taiwan, R.O.C. E-mail: wjsu@vghtpe.gov.tw

Received: June 112013 | Accepted after revision: Sept 022013 | First published online: Sept 262013

Support statement: This study was funded by the Institute for Biotechnology and Medicine Industry, Taiwan, R.O.C., and Taipei Veterans General Hospital (V100A-002, V101B-027 and V102B-030).

Conflict of interest: None declared.

\title{
References
}

1 Barry CE 3rd, Boshoff HI, Dartois V, et al. The spectrum of latent tuberculosis: rethinking the biology and intervention strategies. Nat Rev Microbiol 2009; 7: 845-855.

2 Garmendia J, Morey P, Bengoechea JA. Impact of cigarette smoke exposure on host-bacterial pathogen interactions. Eur Respir J 2012; 39: 467-477.

3 Leung CC, Yew WW, Law WS, et al. Smoking and tuberculosis among silicotic patients. Eur Respir J 2007; 29: 745-750.

4 den Boon S, van Lill SWP, Borgdorff M, et al. Association between smoking and tuberculosis infection: a population survey in a high tuberculosis incidence area. Thorax 2005; 60: 555-557.

5 Horne DJ, Campo M, Ortiz JR, et al. Association between smoking and latent tuberculosis in the U.S. population: an analysis of the National Health and Nutrition Examination Survey. PLoS One 2012; 7: e49050.

6 Diel R, Loddenkemper R, Zellweger JP, et al. Old ideas to innovate tuberculosis control: preventive treatment to achieve elimination. Eur Respir J 2013; 42: 785-801.

7 Shang S, Ordway D, Henao-Tamayo M, et al. Cigarette smoke increases susceptibility to tuberculosis - evidence from in vivo and in vitro models. J Infect Dis 2011; 203: 1240-1248.

8 Oni T, Gideon HP, Bangani N, et al. Smoking, BCG and employment and the risk of tuberculosis infection in HIVinfected persons in South Africa. PLoS One 2012; 7: e47072.

9 Aabye MG, Hermansen TS, Ruhwald M, et al. Negative effect of smoking on the performance of the QuantiFERON TB gold in tube test. BMC Infect Dis 2012; 12: 379 .

10 World Health Organization. The International Union Against Tuberculosis and Lung Disease. A WHO/The Union Monograph on TB and Tobacco Control. Joining Efforts to Control Two Related Global Epidemics. Geneva, WHO, 2007; p. 9.

\section{A tale of two settings: the role of the Beijing genotype in the epidemiology of multidrug-resistant tuberculosis}

\section{To the Editor:}

Described as a "template for success" by НАNEKOM et al. [1] the Beijing genotype of Mycobacterium tuberculosis has been associated with hypervirulence, drug resistance, evasion of the bacille Calmette-Guérin (BCG) vaccine and differential immunoregulation [1]. The genotype is itself diverse; accordingly the fact that specific traits have been associated with Beijing only in certain settings may be explained by the variation in the subtypes that predominate in each. 
We sought to explore the role that the Beijing genotype plays in the epidemiology of multidrug-resistant (MDR) tuberculosis (TB) in two very different epidemiological settings: England and Peru. In the UK nearly three quarters of TB disease occurs amongst migrants, which may result from latent M. tuberculosis infections acquired from high-burden countries of origin. Thus the distribution of lineages in the UK reflects a composite of the circulating strains in other nations. Whilst increasing levels of MDR-TB in the UK have triggered understandable concern, only a small fraction of cases of TB are MDR (in 2011 this was $1.0 \%$ ) [2]. In contrast, the Peruvian TB epidemic is relatively "home-grown" and the prevalence of drug resistance is greater (in $20115.3 \%$ were MDR-TB) [2]. Our objectives were thus: 1) identify any association between the Beijing genotype and MDR in these two settings, and 2) to examine if trends in Beijing might explain changes in the prevalence of MDR-TB over time.

Four datasets were used during this study: two English studies and two Peruvian studies.

The English datasets were taken from Public Health England's Enhanced Tuberculosis Surveillance System and Mycobacterial Surveillance Network and were referred to as England 1 and England 2. England 1 consisted of 8859 individuals diagnosed and 24-loci MIRU-VNTR (mycobacterial interspersed repetitive units variable number tandem repeats) strain-typed between 2010 and 2011. Of these, 4661 had at least first-line drug sensitivity data, a minimal 12-loci predefined profile [3] and were pulmonary. England 2 consisted of 189 MDR-TB cases from 2004 to 2007, which had undergone 24-loci typing. 117 were pulmonary and has at least the minimal standard 12-loci profile. 24-loci typing was not available for nonMDR cases during this period. Susceptibility to rifampicin, isoniazid, ethambutol, pyrazinamide, streptomycin, amikacin, kanamycin, capreomycin, azithromycin, clarithromycin, ciprofloxacin, ofloxacin,

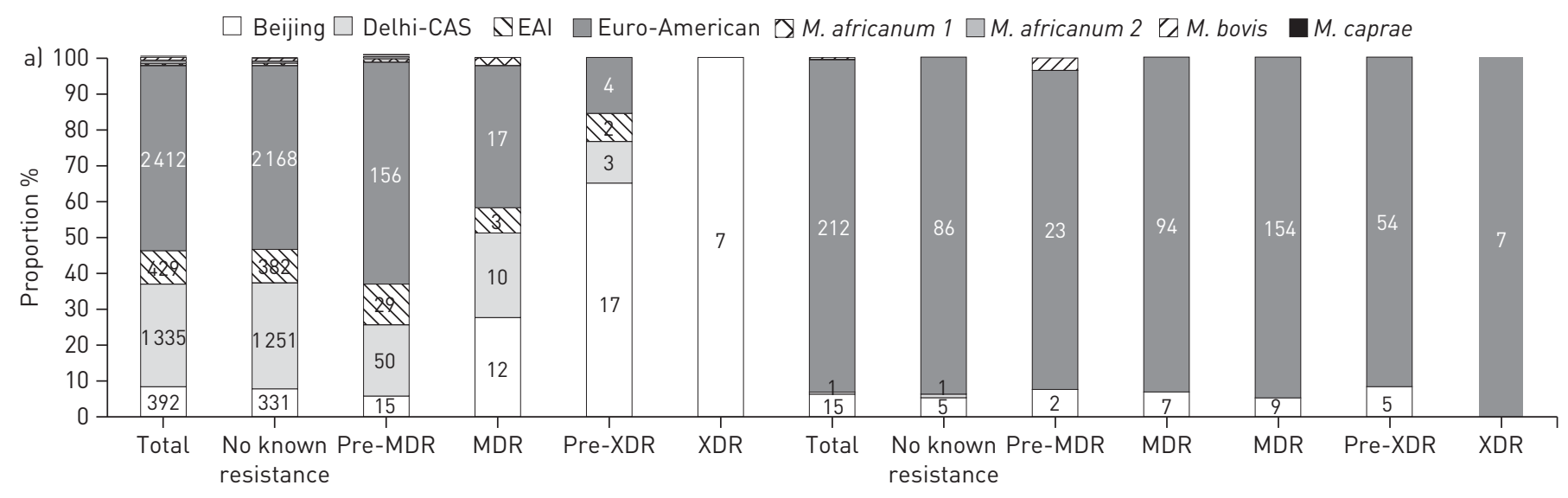

Mutually exclusive resistance categories

England 1

Peru 1

Peru 2

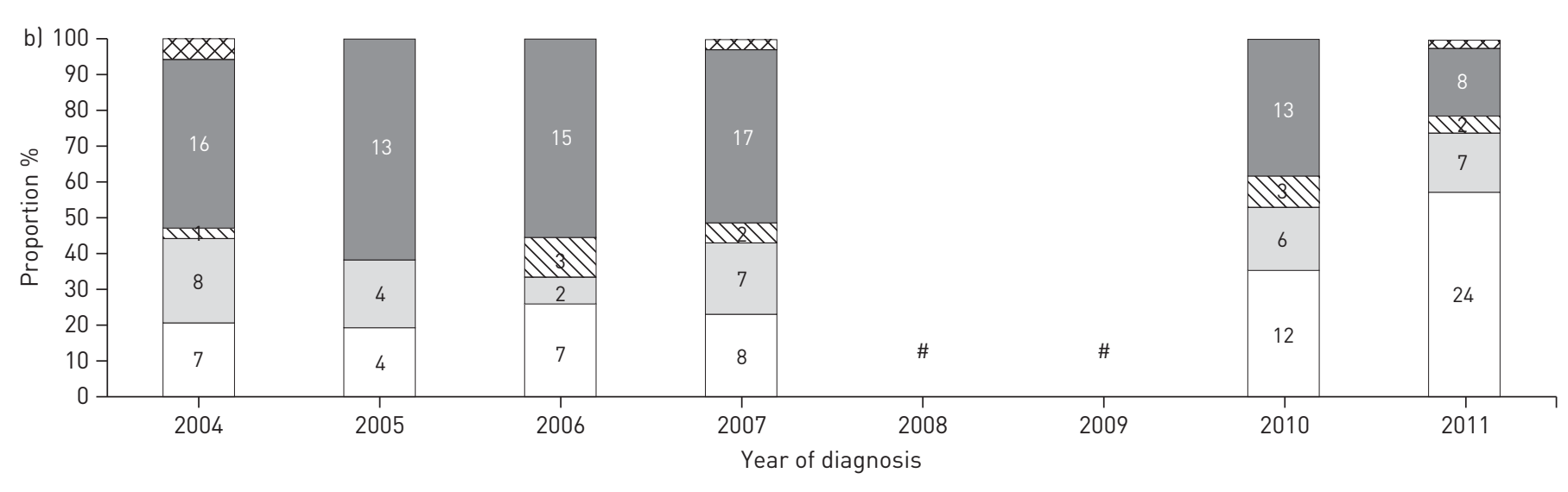

England 1

England 2

FIGURE 1 a) The Beijing genotype is enriched with increasing drug resistance in English (England 1) (ptrend $<0.005$ Bejing versus non-Beijing), but not Peruvian (Peru 1 and Peru 2) pulmonary tuberculosis (TB) cases. b) Lineages of the English pulmonary multidrug resistant (MDR) TB cases (England 1 and England 2), presented by year of diagnosis. The proportion of cases associated with the Beijing genotype may be increasing with time in the latter years. CAS: central Asian; M.: Mycobacterium; EAI: East-African Indian; XDR: extensively drug resistant. *: 24-loci MIRU-VNTR typing was not undertaken between 2008 and 2009. 
moxifloxacin, prothionamide, ethionamide, cycloserine, 4-aminosalicylic acid, linezolid, clofazamine and rifabutin had been tested using the proportion or resistance ratio methods.

The Peruvian datasets were referred to as Peru 1 and Peru 2. Peru 1 consisted of 323 sputum-positive cases of pulmonary TB, recruited from clinical facilities in Lima between 2004 and 2006 and extracted from TAYPE et al. [4]. 229 had been tested for sensitivity to rifampicin, isoniazid, ethambutol and streptomycin, of which $101(44.1 \%)$ were MDR; we note that this figure is much higher than the overall proportion of MDR in this setting [2], suggesting that drug susceptibility testing was prioritised for those at highest risk of MDR, consistent with previous Peruvian testing policies. All cases had a complete standard 12-loci profile. Peru 2 consisted of 230 cases of pulmonary MDR-TB identified in Lima between 1996 and 2004 [5, 6]. Isolates underwent drug susceptibility testing to rifampicin, isoniazid, ethambutol, pyrazinamide, streptomycin, kanamycin, capreomycin, ethionamide, 4-aminosalicylic acid and levofloxacin. One individual lacking a complete standard 12-loci profile was excluded, leaving 229.

MIRU-VNTRplus was utilised to designate lineages for all four datasets to ensure comparability [7]. Substantial differences in the lineage and sublineage profiles of English (England 1, 4661 individuals) and Peruvian (Peru 1, 229 individuals) pulmonary TB cases were apparent (Chi-squared test of independence pvalue $<0.005)$. The vast majority of Peruvian cases were of the Euro-American lineage $(212(92.6 \%)$ out of 229), with Latin American-Mediterranean (LAM) the majority sublineage (102 (48.1\%) out of 212). The remainder of cases were nearly all Beijing (15 (6.6\%) out of 229). In contrast, English cases comprised of a variety of lineages consistent with their multinational origins: Euro-American $2412(51.8 \%)$ out of 4661, Delhi-CAS (central-Asia) 1335 (28.6\%) out of 4661, East African Indian (EAI) 429 (9.2\%) out of 4661, Beijing 392 (8.3\%) out of 4661. The Euro-American sublineages observed were also diverse.

Restricting our analysis to MDR cases revealed very different profiles between Peru and England ( $\mathrm{p}$-value $<0.005)$. Peruvian cases (Peru 2, 229 individuals) were dominated by the Euro-American lineage (215 (93.9\%) out of 229), of which 121 (56.3\%) out of 215 was the LAM sublineage. Regression modelling of Peru 1 to explore the relationship between MDR and M. tuberculosis lineage showed no association (Beijing crude OR (95\% CI) 1.10 (0.38-3.14), Wald p-value 0.86; Euro-American baseline). In stark contrast to the overall distribution of lineages, among English MDR cases (England 1) the proportion Beijing increased to 36 (47.4\%) out of 76. Regression modelling revealed a strong positive association between Beijing and MDR among English pulmonary cases, (crude OR 11.51 (6.65-19.95), p<0.005; adjusted OR 9.53 (4.87-18.65), $\mathrm{p}<0.005$; Euro-American baseline and England 1 used for both models; models excluded non- $M$. tuberculosis cases and those with missing data). Age, sex, a composite social risk factor variable (homelessness, imprisonment, drug/alcohol abuse), country of birth, ethnic group and previous diagnosis were adjusted for in the latter; this could not be done for Peru 1 as these variables were not available.

We also tested whether Beijing was enriched among strains with resistance to a greater number of drugs (fig. 1a). Pre-MDR-TB (pre-MDR) was defined as resistance to either, but not both, isoniazid and rifampicin. Pre-extensively drug resistant (pre-XDR) cases were MDR plus resistant to either a fluoroquinolone or a second-line injectable. Beijing was enriched from pre-MDR to XDR cases in the English data (Pearson's correlation coefficient (ptrend) Beijing versus non-Beijing $\mathrm{p}<0.005$; England 1 ). This could be specifically observed among non-UK born, but not UK born, cases (data not shown). No trend was seen in the Peruvian data (ptrend=0.76; Peru 1 and Peru2). Further analysis to determine whether the association has changed over time indicated a recent increase in the proportion of cases caused by Beijing (ptrend $<0.005$; England 1 and England 2) (fig. 1b).

Our analysis suggests that the Beijing genotype plays a different role in the current epidemiology of MDRTB in England and Peru. In England, the observed association likely reflects the rising importation of resistant strains, rather than the development of resistance within the UK. In Peru there is likely to be a similarly high prevalence of MDR among all genotypes, potentially explaining why we did not observe an association. Interestingly, a recent paper by IШАмото et al. [8] suggests that the overall prevalence of Beijing has increased in Peru over the last decade, but the proportion of Beijing strains that are MDR has decreased.

We utilised available datasets from studies undertaken at different times with variable designs, thus some interpretative caution is required. For example, Peru 2 recruited from households with more than one pulmonary MDR case, and thus could have oversampled clustered cases. However, the similarity of the lineage profiles of Peru 2 with Peru 1, which was not collected through such means, suggests this effect is limited. English cases were tested for resistance to a wider range of drugs than the Peruvian cases, thus the "no known resistance" category is not strictly comparable between the two datasets. Nevertheless, only 1.3\% of the England 1 dataset was resistant to drugs not tested within the Peru 1 dataset.

Molecular epidemiological surveillance and addressing and preventing resistance have been highlighted as vital for TB elimination by the European Respiratory Society $[9,10]$. We demonstrate within this study how 
the tools of the former could be vital to aid the latter: within Peru the MDR epidemic could worsen should fitter drug resistant Beijing strains evolve or enter the country and within the UK where the lineage profile is highly dynamic.

Contrasting relationship between the Beijing genotype of Mycobacterium tuberculosis and MDR-TB in England versus Peru. http://ow.ly/qjxTG

Helen R. Stagg ${ }^{1,2,3}$, Ted Cohen ${ }^{3,4}$, Mercedes C. Becerra ${ }^{5}$, Laura F. Anderson ${ }^{2}$ and Ibrahim Abubakar ${ }^{1,2}$

${ }^{1}$ Research Dept of Infection and Population Health, University College London, and ${ }^{2}$ TB Section, Dept of Respiratory Diseases, Public Health England, London, UK. ${ }^{3}$ Division of Global Health Equity, Brigham and Women's Hospital, Boston, MA, ${ }^{4}$ Dept of Epidemiology, Harvard School of Public Health, Boston, MA, and ${ }^{5}$ Dept of Global Health and Social Medicine, Harvard Medical School, Boston, MA, USA.

Correspondence: H.R. Stagg, Research Dept of Infection and Population Health, University College London, 4th floor, Mortimer Market Centre, Mortimer Market, London, WC1E 6JB, UK. E-mail: h.stagg@ucl.ac.uk

Received: July 032013 | Accepted after revision: Aug 312013 | First published online: Sept 132013

Support statement: H.R. Stagg was supported by a European Respiratory Society Fellowship STRTF 24-2011. The strain typing of the English datasets was funded by Public Health England's Strain Typing Project. I. Abubakar was funded through an NIHR Senior Research Fellowship NIHR-2011-04-001.

Conflict of interest: Disclosures can be found alongside the online version of this article at www.erj.ersjournals.com

Acknowledgements: We acknowledge our utilisation of the published data of TAYPE et al. [4]. Our study made use of the MIRU-VNTRplus database website (www.miru-vntrplus.org) developed by D. Harmsen, S. Niemann, P. Supply and T. Weniger.

\section{References}

1 Hanekom M, Gey van Pittius NC, McEvoy C, et al. Mycobacterium tuberculosis Beijing genotype: a template for success. Tuberculosis (Edinb) 2011; 91: 510-523.

World Health Organization. Global Tuberculosis Control Report. Geneva, World Health Organization, 2012.

3 Savine E, Warren RM, van der Spuy GD, et al. Stability of variable-number tandem repeats of mycobacterial interspersed repetitive units from 12 loci in serial isolates of Mycobacterium tuberculosis. J Clin Microbiol 2002; 40: 4561-4566.

4 Taype CA, Agapito JC, Accinelli RA, et al. Genetic diversity, population structure and drug resistance of Mycobacterium tuberculosis in Peru. Infect Genet Evol 2012; 12: 577-585.

5 Becerra MC, Appleton SC, Franke MF, et al. Tuberculosis burden in households of patients with multidrugresistant and extensively drug-resistant tuberculosis: a retrospective cohort study. Lancet 2011; 377: 147-152.

6 Mitnick C, Bayona J, Palacios E, et al. Community-based therapy for multidrug-resistant tuberculosis in Lima, Peru. N Engl J Med 2003; 348: 119-128.

7 Allix-Béguec C, Harmsen D, Weniger T, et al. Evaluation and strategy for use of MIRU-VNTRplus, a multifunctional database for online analysis of genotyping data and phylogenetic identification of Mycobacterium tuberculosis complex isolates. J Clin Microbiol 2008; 46: 2692-2699.

8 Iwamoto T, Grandjean L, Arikawa K, et al. Genetic diversity and transmission characteristics of Beijing family strains of Mycobacterium tuberculosis in Peru. PLoS One 2012; 7: e49651.

9 Blasi F, Reichman LB, Migliori GB. Presenting the European Forum for TB Innovation: innovative thinking in progressing towards TB elimination in Europe. Eur Respir J 2012; 40: 806-808.

10 Diel R, Loddenkemper R, Zellweger JP, et al. Old ideas to innovate tuberculosis control: preventive treatment to achieve elimination. Eur Respir J 2013; 42: 785-801.

\section{Helicobacter pylori seroprevalence in patients with idiopathic pulmonary fibrosis}

\section{To the Editor:}

In 2007, the European Respiratory Journal published a letter by IBRAHIM [1] in which it was suggested that micro-aspiration of Helicobacter pylori from gastric juice secondary to gastro-oesophageal reflux disease (GORD) may cause or contribute to the development of idiopathic pulmonary fibrosis (IPF) through recurrent lung insult. This hypothesis was derived from the observation that GORD is common in IPF patients $[2,3]$, while in the general population the prevalence of GORD exceeds that of IPF. IBRAHIM [1] 